\title{
Sigmoid colon vaginoplasty in a Mayer-Rokitansky-Kuster-Hauser (MRKH) syndrome patient with situs inversus totalis and review.
}

\author{
Apurva Bhaskar ${ }^{1}$, Sommya Gopinath ${ }^{2}$, and Subramanya Kattepura ${ }^{3}$ \\ ${ }^{1}$ Affiliation not available \\ ${ }^{2}$ District hospital tumkur \\ ${ }^{3}$ Sri Siddhartha Academy of Higher Education
}

April 28, 2020

\begin{abstract}
A 26 years old lady presented to us with primary amenorrhoea. On examination and investigation she was found to have MRKH syndrome with Situs inversus totalis. Likewise what we do for these syndromic patients, we did sigmoid vaginoplasty. This is one of the uncommon anatomical variants we encountered in our series of cases. Our literature search did not reveal such patient undergoing sigmoid vaginoplasty and so is this case presented for publication.
\end{abstract}

Introduction:

Sigmoid vaginoplasty is a common procedure done for patients with MRKH syndrome with its known benefits. However, situs inverses totalis with MRKH is a rare entity. We discuss the sigmoid vaginoplasty done on this patient and the follow up. This case is presented here to bring to light of this unique combination of genital and gastrointestinal anomalies and its management.

Case capsule:

A 26 years old lady presented to us with primary amenorrhoea. She had her first consultation for the same problem at the age of 16 years. An ultrasound was done showed absent uterus and normal ovaries. She was advised a Magnetic Resonance (MR) imaging for further clarification. At that point, she could not get this done due to financial and social circumstances.

On examination, she had fully developed secondary sexual characters. Perineal examination revealed a shallow pit in the place of vagina.

Karyotyping was 46XX; FSH and LH levels were normal.

A MR imaging done now showed transposition of intra abdominal viscera and dextrocardia - situs inversus totalis, horse shoe kidney and absent left ovary and the uterus (Fig 1).

As the patient has situs inversus totalis, we decided to do an arteriogram to look for the inferior mesenteric arterial arcade. The vascular pattern was an exact mirror image of the normal pattern (Fig 2).

After thorough counselling with the patient and the parents, we prepared her bowel and took her for bowel vaginoplasty. A vertical midline below umbilical incision was made. The pelvis was inspected - there was normal looking right ovary above the pelvic brim and the left ovary was absent. The uterus was rudimentary. A tunnel was created in the recto-vesical channel by simultaneous dissection in perineum and the vaginal pit. The track was dilated using the Hegar's dilater. The descending colon was mobilised from the splenic 
flexure. The sigmoid colon was severed beneath the left colic artery and at the level of the pelvic brim (Fig 3). Few proximal sigmoid vessels supplying graft were tied to gain more length. The distal end of the sigmoid colon was closed using the absorbable sutures. The proximal end now rotated at 180 degrees on the sigmoid vascular pedical (inverted pedicles graft). The open proximal end is brought out of the vaginal pit in the Introits and anastomosed. The descending colon was anastomosed to the rectum after mobilisation of splenic flexure and the abdomen was closed. The post-operative period was uneventful. She was started on feeds from the 4th day of surgery and was discharged on the 6th day of surgery. She was started on dilatation on the following week. Vaginal douching was advised once a day. She is on regular follow-up with no other complaints; her vagina admits two fingers and is about $10 \mathrm{cms}$ long. She is yet to find a partner.

Discussion:

Mayer-Rokitansky-Kuster-Hauser (MRKH) syndrome is a rare congenital anomaly with a genotype of 46XX. Here we see aplasia of the uterus and vagina with normal ovaries and normal development of secondary sexual characteristics. MRKH presents in two types. Type 1 (Rokitansky sequence) is Mullarian duct aplasia in isolation. Type 2 or MURCS (Mullarian renal cervical somite) association. The MURCS association has triad of main manifestations - genital, renal (unilateral absent kidneys, ectopic kidneys, horseshoe kidneys), and skeletal defects (kypho-scoliosis, fused cervical vertebrae, stapedial ankylosis) ${ }^{1}$. MRKH type 2 is thought to be the more severe form of the MRKH spectrum ${ }^{2}$. This disease when occurs in familial settings, is inherited as Autosomal dominant pattern. The disease presents with incomplete penetrance and variable expressivity. The situs inversus totalis is seen in MRKH type 2 or MURCS association ${ }^{2}$. These defects suggest the involvement of the homeobox (HOX) genes. HOX genes play a key role during the embryogenesis of axial skeleton, hind brain and urogenital system ${ }^{3}$. However, we could not find skeletal association in our patient.

The situs inversus totalis is also called the "mirror man", where the internal organs of body are rotated by 180 degrees. It is called "totalis" when even the heart is on the right side. Surgical diagnosis and surgical procedures in these patients is challenging due to the mirror image anatomy. This degree of difficulty is more pronounced while doing laparoscopic surgeries. Other important aspect of situs inversus is the associated vascular anomalies ${ }^{4}$. This made us to do the angiography in our patient to have a prior vascular anatomy knowledge. Intra - operatively, the vascular arcades looked better from the left side of the patient. We did not encounter any problems during the procedure.

Very important aspect of treatment of MRKH is age of the patient. Treatment of MRKH is a multidepartmental approach.

The non-operative Frank and Ingram technique of progressive pressure onto the vaginal pit using dilators has been less popular due to its unsatisfactory results and poor compliance ${ }^{5}$.

Many operative (McIndoe's, William's, Vecchieti's,) methods of vaginal replacement have been explained, McIndoe's technique, where the skin grafts are placed between the urethra and the rectum by blunt dissection in this area. William's vaginoplasty involves a vulvar flap to make the vaginal tube. The Vecchieti technique is based on stretching of the vaginal dimple via acrylic mould passed in the neo-vaginal space and pulled by sub-peritoneal threads. The vagina will lengthen over a period of days to weeks ${ }^{5}$.

The sigmoid vaginoplasty has stood the test of time ${ }^{1}$. The disadvantage with this technique is its preoperative preparation, invasiveness and bowel complications like anastomotic leak, mechanical bowel obstruction, and diversion colitis ${ }^{5}$. Definitely, there is nothing to match characters of the normal vagina; however sigmoid colon has many similarities compared to normal vagina. The sigmoid colon can be harvested either in isoperistaltic or anti peristaltic manner. Few studies have also shown the advantages of ileum over sigmoid colon - no feculant smell and diversion colitis ${ }^{5}$. The diversion colitis is a problem in colonic diversions due to lack of short chain fatty acids and colonic bacteria of faeces in the diverted segment ${ }^{5}$.

Selecting the part of the intestine (Ilium vs Sigmoid) is more to do with surgeons comfort and familiarity with the procedure. 
To avoid injury to the rectum and the urinary bladder - we start the abdominal dissection first by following the utero-sacral ligaments to the rudimentary uterus. Then the vaginal dissection started.

Laparoscopic sigmoid vaginoplasty has its own advantages like better cosmetic results, less invasiveness and early recovery. However, the disadvantage of Laparoscopy being long learning curve and long operating time $^{5}$. Various methods have been explained on harvesting the sigmoid graft based on the vascular pedicle; what we did was the inverted sigmoid colon graft based on sigmoid arteries.

The other disadvantage of sigmoid vaginoplasty is prolonged periods of fasting due to bowel preparation before surgery and bowel anastomosis. Recently many centers are using Enhanced Recovery After Surgery (ERAS) protocols - fast track bowel regimes have been used for early recuperation from this major surgery ${ }^{6}$.

In rare instances, MRKH mothers can have children with their oocytes being harvested, fertilised and implanted in a surrogate mother. It is also important that these mothers should take the decision to conceive seriously, as this is of genetic origin.

Conclusion:

MRKH with Situs inversus totalis is a rare congenital anomaly. Sigmoid vaginoplasty is the gold standard procedure. Operating on this unique combination of anomalies needs prior vascular anatomy knowledge for mental orientation.

Disclosure of interests: None

Contribution of authorship: This write up is a teamwork. The main author of this paper is Dr Subramanya Kattepura, who is also the chief surgeon and he was assisted is various aspects by the other two authors Drs Apurva Bhaskar and Sowmya Gopinath.

Patient's consent: We have taken a written informed consent of the patient.

We have not received any funding for this paper.

References:

1. Pai A, Shakir M. Mayer-Rokitansky-Kuster-Hauser syndrome type II: A rare case. Indian J Hum Genet. 2013 Jan;19(1):113-5.

2. Alka V. Ekbote, Mohan S. Kamath, Sumita Danda. MURCS association with situs inversus totalis: Expanding the spectrum or a novel disorder. J Pediatr Genet. 2014 Sep; 3(3): 167-173.

3. Mungadi IA, Ahmad Y, Yunusa GH, Agwu NP, Ismail S. Mayer-rokitansky-kuster-hauser syndrome: surgical management of two cases. J Surg Tech Case Rep. 2010 Jan;2(1):39-43.

4. Deng Xiang, Jiannan He, Zimeng Fan, Fangfang Xiong, Gang Liu, Sufen Chen, Wu Wen, Jianfeng Li, Junhua Ai, Renhua Wan, Gongxian Wang, Jun Shi. Situs inversus totalis with solid pseudopapillary pancreatic tumor: A case report and review of literature. Medicine (Baltimore) 2018 Mar; 97(12): e0205. Published online 2018 Mar 23.

5. L.Al-Mehaisen, Z.Amarin, O.Bani Hani, F.Ziad, O.Al-Kuran. Ileum neovaginoplasty for Mayer-RokitanskyKüster-Hauser: Review and case series. African Journal of Urology. Vol 23, No 2 (2017)

6. Megan Melnyk, Rowan G. Casey, Peter Black, Anthony J. Koupparis, Enhanced recovery after surgery (ERAS) protocols: Time to change practice? Can Urol Assoc J. 2011 Oct; 5(5): 342-348.

Legends for figures:

Figure 1: MRI of the abdomen and thorax showing situs inversus totalis, horseshoe pelvic kidney and the left ureter.

Figure 2: CT aortogram showing the mirror image of the vascular architecture. The left colic artery and sigmoid arteries, the branch of Inferior mesenteric artery are on the right side. 
Figure 3: Sigmoid colon graft based on the simoid vessels is ready for pull through as neo-vagina.

\section{Hosted file}

Figure 1.docx available at https://authorea.com/users/307137/articles/438107-sigmoid-colon-vaginoplastyin-a-mayer-rokitansky-kuster-hauser-mrkh-syndrome-patient-with-situs-inversus-totalis-andreview

\section{Hosted file}

Figure 2.docx available at https://authorea.com/users/307137/articles/438107-sigmoid-colon-vaginoplastyin-a-mayer-rokitansky-kuster-hauser-mrkh-syndrome-patient-with-situs-inversus-totalis-andreview

\section{Hosted file}

Figure 3.docx available at https://authorea.com/users/307137/articles/438107-sigmoid-colon-vaginoplastyin-a-mayer-rokitansky-kuster-hauser-mrkh-syndrome-patient-with-situs-inversus-totalis-andreview 